\title{
An Entamoeba histolytica rhomboid protease with atypical specificity cleaves a surface lectin involved in phagocytosis and immune evasion
}

\author{
Leigh A. Baxt, ${ }^{1}$ Rosanna P. Baker, ${ }^{2}$ Upinder Singh, ${ }^{1,4}$ and Sinisa Urban ${ }^{2,3}$ \\ ${ }^{1}$ Departments of Internal Medicine and Microbiology and Immunology, Stanford University School of Medicine, Stanford, \\ California 94305, USA; ${ }^{2}$ Department of Molecular Biology and Genetics, Johns Hopkins University School of Medicine, \\ Baltimore, Maryland 21205, USA
}

\begin{abstract}
Rhomboid proteases are membrane-embedded enzymes conserved in all kingdoms of life, but their cellular functions across evolution are largely unknown. Prior work has uncovered a role for rhomboid enzymes in host cell invasion by malaria and related intracellular parasites, but this is unlikely to be a widespread function, even in pathogens, since rhomboid proteases are also conserved in unrelated protozoa that maintain an extracellular existence. We examined rhomboid function in Entamoeba histolytica, an extracellular, parasitic ameba that is second only to malaria in medical burden globally. Despite its large genome, $E$. histolytica encodes only one rhomboid (EhROM1) with residues necessary for protease activity. EhROM1 displayed atypical substrate specificity, being able to cleave Plasmodium adhesins but not the canonical substrate Drosophila Spitz. We searched for substrates encoded in the ameba genome and found EhROM1 was able to cleave a cell surface lectin specifically. In E. histolytica trophozoites, EhROM1 changed localization to vesicles during phagocytosis and to the posterior cap structure during surface receptor shedding for immune evasion, in both cases colocalizing with lectins. Collectively these results implicate rhomboid proteases for the first time in immune evasion and suggest that a common function of rhomboid enzymes in widely divergent protozoan pathogens is to break down adhesion proteins.
\end{abstract}

[Keywords: Serine protease; presenilin; site-2 protease; erythrophagocytosis; immune evasion; pathogenesis]

Received February 22, 2008; revised version accepted April 17, 2008.

Studying widely conserved regulatory proteins in organisms that are not amenable to genetic dissection is a valuable post-genomic strategy for identifying factors that regulate important cellular processes. Rhomboid proteases are multitransmembrane proteins with a serine protease active site embedded within the membrane (Urban et al. 2001; Wang et al. 2006; Wu et al. 2006; Ben-Shem et al. 2007; Lemieux et al. 2007). These unusual enzymes are rare among membrane proteins in being conserved throughout evolution in all kingdoms, but their cellular roles remain poorly understood (Koonin et al. 2003; Urban 2006). Rhomboid was first discovered in the model metazoan Drosophila, where it functions in cleaving epidermal growth factor (EGF) ligands to activate receptor tyrosine kinase signaling during development (Bier et al. 1990; Urban et al. 2002a). Initial mechanistic analysis with Rhomboid-1 from Drosophila revealed that it recognizes its substrate, the EGF ligand

Corresponding authors.

${ }^{3}$ E-MAIL surban@jhmi.edu; FAX (410) 502-3178.

${ }^{4}$ E-MAIL usingh@stanford.edu; FAX (650) 724-3892.

Article is online at http://www.genesdev.org/cgi/doi/10.1101/gad.1667708.
Spitz, by the top of the Spitz transmembrane segment (Urban and Freeman 2003). This region is composed of helix-breaking residues that are essential for cleavage, and probably facilitate substrate unwinding from the membrane into the internal active site of the enzyme (Baker et al. 2007). Rhomboid enzymes from many organisms are able to cleave Spitz and in doing so rely on this sequence in Spitz (Urban and Freeman 2003; Urban and Wolfe 2005). One specialized subclass of rhomboid enzymes is known to be imported into mitochondria, where it functions in mitochondrial membrane dynamics (Herlan et al. 2003; McQuibban et al. 2003; Cipolat et al. 2006; Jeyaraju et al. 2006).

Little is known about rhomboid enzyme function in most eukaryotes, especially unicellular eukaryotes. A broad homology search for the transmembrane motif recognized by Drosophila Rhomboid-1 identified a similar sequence within adhesins that mediate invasion of host cells by Apicomplexan parasites, including Toxoplasma, Cryptosporidium, and Plasmodium (Urban and Freeman 2003). These transmembrane adhesins bind host cell receptors during invasion, but ultimately their cleavage is essential to release the junctions and facilitate parasite 
internalization into the host cell (Carruthers et al. 2000). The cleavage site of several adhesins has been mapped to their transmembrane segments, and rhomboid enzymes are thought to provide this cleavage event (Opitz et al. 2002; Howell et al. 2005; O'Donnell et al. 2006). Toxoplasma gondii rhomboid 5 (TgROM5) can cleave the canonical rhomboid substrate Spitz and is localized to the posterior surface of the tachyzoite, where it cleaves parasite adhesins facilitating host invasion (Brossier et al. 2005; Dowse et al. 2005). In Plasmodium, a homolog of TgROM5 is missing, but two rhomboid enzymes, Plasmodium falciparum rhomboid 1 (PfROM1) and Plasmodium falciparum rhomboid 4 (PfROM4), appear to compensate functionally (Baker et al. 2006). The Plasmodium rhomboid that is specialized for cleaving Spitztype substrates is PfROM1. Conversely, PfROM4 was the first rhomboid enzyme discovered that cannot cleave Spitz, yet is able to process most Plasmodium adhesins (Baker et al. 2006; O'Donnell et al. 2006). Importantly, most Plasmodium adhesins cannot be cleaved by nonApicomplexan rhomboid enzymes (Baker et al. 2006). The evolutionary and functional significance of this new mode of specificity is unclear, but warrants further investigation.

Despite these advances, breakdown of junctions during invasion is unlikely to be a common function for rhomboid enzymes, even among protozoa, since most eukaryotic cells maintain an extracellular existence. To address what might be a more general function for rhomboid proteases among widely diverse eukaryotic pathogens, we investigated rhomboid function in Entamoeba histolytica, a parasitic ameba that is rare among eukaryotes in that it encodes only one rhomboid enzyme. Unlike Apicomplexa, E. histolytica is an extracellular protozoan parasite. It infects $\sim 500$ million people per year, 50 million of whom develop invasive disease (Ackers et al. 1997). The parasite exists in two forms, the dormant cyst and the invasive trophozoite form. Invasive disease occurs when trophozoites adhere to the colonic epithelium, lyse the intestinal cells, and penetrate deeply into colonic tissues (Stanley 2003). Some factors that are important in amebic pathogenesis have been identified, including the Gal/GalNAc surface lectin (important in host cell adhesion and induction of apoptosis), amebapores (pore-forming proteins important in lysis of colonic bacteria), and cysteine proteases (degrade mucin, extracellular matrix, IgA, and IgG and activate complement) (for review, see Stanley 2003). Other determinants of amebic virulence are phagocytosis and motility (Rodriguez and Orozco 1986; Coudrier et al. 2005).

In order to colonize the host successfully, the ameba must evade the host immune system. A unique mechanism that $E$. histolytica uses for immune evasion is surface receptor capping, during which surface receptors that have been recognized by host immune components are rapidly polarized to the posterior of the cell and released, essentially shedding the parasite surface (Calderon 1980; Espinosa-Cantellano and Martinez-Palomo 1994). Despite the importance of these processes, little is known about how they are achieved at the molecular level, partly because E. histolytica is not amenable to genetic dissection.

We have investigated the function of the conserved rhomboid family of proteases in E. histolytica, which remarkably encodes only one rhomboid protein (termed EhROM1) with the residue requirements for proteolytic activity. Surprisingly, EhROM1 displayed the newly discovered, atypical mode of substrate specificity analogous to PfROM4. A candidate substrate search revealed that EhROM1 is able to cleave an ameba lectin, which are generally known to play key roles in ameba pathogenesis. In amebic trophozoites, EhROM1 changed localization to colocalize with a lectin in vesicles during phagocytosis and the base of the cap during surface receptor capping. These observations implicate EhROM1 as the first protease discovered for cleaving lectins, which are key for amebic virulence, and uncover a previously unidentified function for rhomboid enzymes in immune evasion.

\section{Results}

Identification of rhomboid genes in E. histolytica

Despite its highly duplicated genome, E. histolytica encodes four rhomboid-like genes but only one that contains the sequence elements known to be required for protease activity (Urban et al. 2001; Loftus et al. 2005). This gene (EAL46300.1), which we have named EhROM1, contains the conserved WR motif in its first extracellular loop, the HxxxxHxxxN sequence on transmembrane (TM) segment 2 that is proposed to be part of the oxyanion-stabilizing pocket, the catalytic serine within the GASG motif on TM4, and the active site histidine within TM6 followed by the universally conserved GxxxG motif. All of the other rhomboid-like genes identified by Pfam searches are missing either the catalytic serine (EAL44183.1), histidine (EAL47860.1), or both (EAL49376.1/EAL51684.1). Moreover, unlike these inactive Entameoba rhomboid-like genes, EhROM1 is highly conserved across both the nonpathogenic Entamoeba dispar and the reptilian Entamoeba invadens and is expressed at high levels in all strains and conditions tested using microarrays (Ehrenkaufer et al. 2007; data not shown). Thus, only one E. histolytica rhomboid, EhROM1, would be expected to function as an intramembrane protease, which is unusual because multiple active rhomboid proteases have been identified in every other eukaryotic organism in which they have been studied (Koonin et al. 2003; Brossier et al. 2005; Dowse and Soldati 2005; Lemberg and Freeman 2007).

\section{Heterologous expression of Entamoeba histolytica ROM1}

In order to assess the proteolytic activity of EhROM1, we attempted to express it in mammalian cells by cotransfection with GFP-tagged Spitz, an insect signaling molecule that serves as a surrogate substrate for most rhomboid enzymes analyzed (Urban et al. 2002b; Urban and Freeman 2003). Transfected mammalian COS cell ly- 

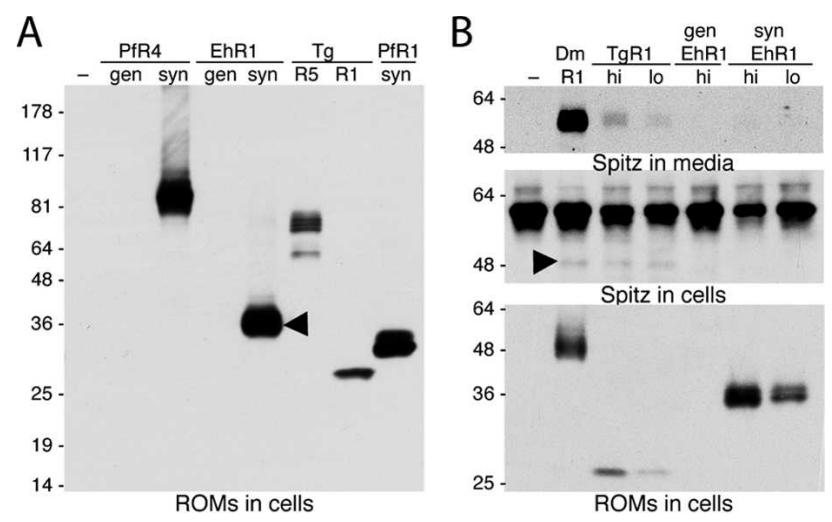

Figure 1. Expression and activity analysis of EhROM1 in transfected mammalian cells. (A) Total cell lysates from transfected COS cells were examined for ROM expression by anti-HA western analysis. Prestained molecular mass standards are indicated in kilodaltons on the left. "Gen" denotes endogenous genomic constructs, while "syn" signifies a codon-optimized synthetic construct used in the transfection. Note that for EhROM1 (EhR1) and control PfROM4 (PfR4), no expression could be detected for genomic constructs, while expression of the synthetic constructs was robust (arrow designates EhROM1). TgR1, TgR5, and PfR1 denote TgROM1, TgROM5, and PfROM1, respectively. (B) Activity analysis of EhROM1 with the canonical substrate Drosophila Spitz as detected by anti-GFP Western of media and cell fractions (cleaved band in cells is denoted with an arrow). While Drosophila Rhomboid-1 (DmR1) and TgROM1 (TgR1) could detectably process Spitz, transfection of neither the genomic nor synthetic expression constructs of EhROM1 resulted in Spitz cleavage. Note that TgROM1 was used as a control because it shows a low level of activity with Spitz, and detection of cleaved Spitz therefore indicated that assay conditions were optimal. "Hi" and "lo" refer to high and low amounts of rhomboid-encoding plasmid DNA used in each transfection, respectively.

sates and media were tested for cleaved Spitz by antiGFP western analysis, since cleaved Spitz is rapidly secreted from the cells into the cell culture media. However, we were unable to detect expression of EhROM1 under a variety of conditions in diverse cell lines including COS, HeLa, HEK 293, and CHO (Fig. 1A; data not shown). We have previously overcome this limitation by recoding PfROM genes, such that the same protein is encoded by a different gene containing optimal codons and sequences for expression in mammalian cells (Baker et al. 2006). Like with PfROM4, recoding the open reading frame of EhROM1 resulted in a dramatic increase of expression from barely detectable to being one of the highest expressed parasite ROMs (Fig. 1A). However, despite high levels of expression, EhROM1 failed to display detectable proteolytic activity against the canonical rhomboid substrate Spitz, while other rhomboid enzymes cleaved Spitz efficiently under identical conditions (Fig. 1B).

\section{EhROM1 cleaves four classes of Plasmodium adhesins}

Recently, we discovered that ROM4 from Plasmodium possesses an atypical mode of substrate specificity in that it is the only rhomboid enzyme known to be unable to cleave Spitz, yet cleaves several classes of adhesins including erythrocyte-binding-like (EBL) and reticulocyte-binding-like (RBL) adhesins (Baker et al. 2006). Interestingly, this specificity is different from canonical rhomboid enzymes studied thus far because these adhesins themselves cannot be cleaved by Spitz-cleaving rhomboid enzymes (Baker et al. 2006). We therefore examined whether EhROM1 could cleave a representative of each of the four families of Plasmodium adhesins: the EBL adhesin BAEBL, the RBL adhesin Rh4, AMA1, and TRAP. These adhesins have completely different sequences in their ectodomains and cytosolic regions, but share some similarities in their transmembrane regions. We discovered that all adhesins were efficient substrates for the recoded EhROM1 with the exception of AMA1, which was cleaved less well than the others by EhROM1 and not at all by PfROM4 (Fig. 2A-D). Without exception, EhROM1 activity against all adhesins depended on the predicted active site serine (Fig. 2A-D). Thus, EhROM1 is an intramembrane protease of the serine class that is able to cleave multiple Plasmodium adhesins and, like PfROM4, cannot cleave the canonical substrate Spitz.

\section{EhROM1 displays distinct substrate specificity analogous to Plasmodium PfROM4}

Rhomboid enzymes typically recognize transmembrane sequences within their substrates to achieve specificity
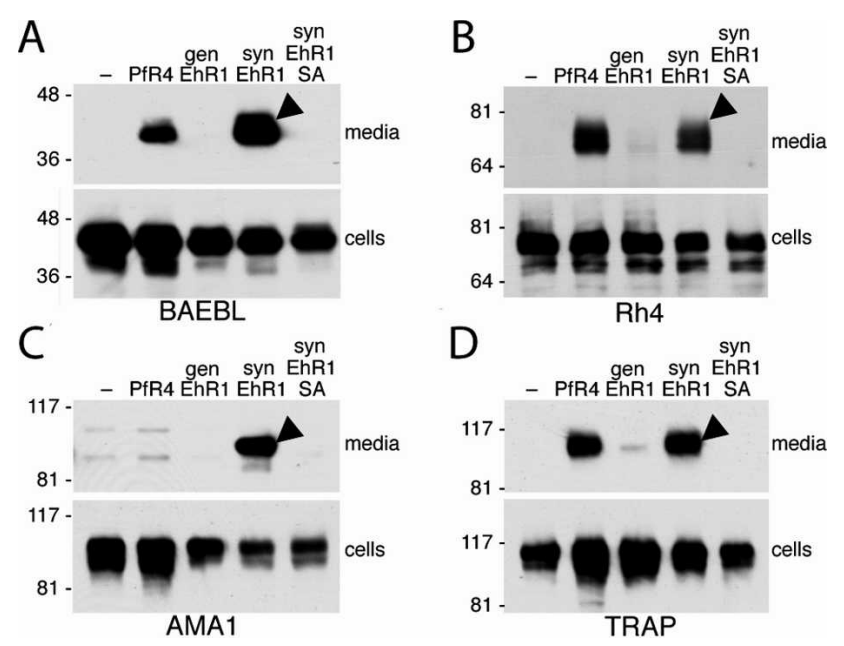

Figure 2. EhROM1 is able to cleave Plasmodium adhesins. GFP-tagged BAEBL $(A), \operatorname{Rh} 4(B), \operatorname{AMA} 1(C)$, and TRAP $(D)$ were cotransfected with EhROM1 constructs or PfROM4 as a positive control. Media and cell fractions were analyzed by anti-GFP Western, with prestained molecular mass standards denoted to the left of each image. EhROM1 expressed from the synthetic construct (synEhR1) was able to process the four Plasmodium adhesins (cleaved bands denoted by arrows), at times better than the corresponding PfROM4 controls. Weak cleavage by the endogenous EhROM1 construct (genEhR1) could also be detected for Rh4 and TRAP. Activity was abolished with a catalytic serine to alanine mutant form of synEhROM1 (syn-EhR1-SA). 
(Urban and Freeman 2003; Baker et al. 2006). To map the sequences required for EhROM1 specificity, we examined its ability to cleave BAEBL and AMA1 adhesins harboring mutations within the top of their transmembrane segments. EhROM1 could not cleave mutated BAEBL or AMA1 adhesins (Fig. 3A,B), indicating that specificity of EhROM1 maps to the upper transmembrane sequence of substrates.

The ability of EhROM1 to cleave Plasmodium adhesins but not Spitz implied that it displays the atypical
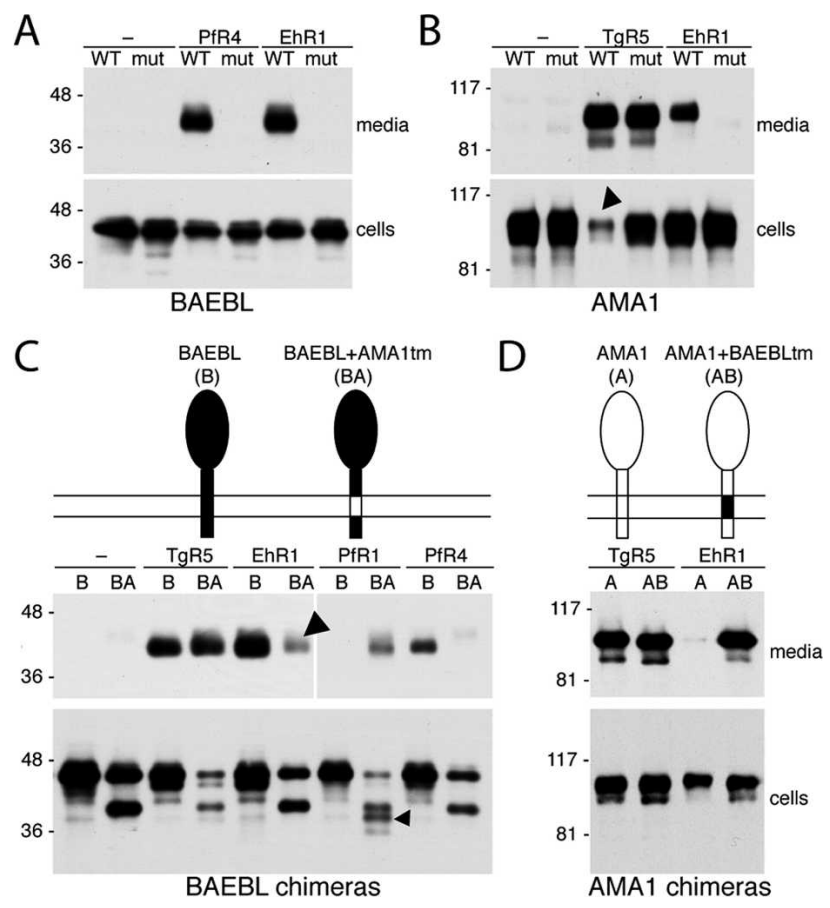

Figure 3. EhROM1 shares specificity with PfROM4 for transmembrane segments. (A) Cleavage of transmembrane mutants of BAEBL $(A)$ and AMA1 $(B)$ were tested with EhROM1. Changing the top of BAEBL's TM segment to that of human TGF $\alpha$, or mutation of the AMA1 TM sequence ASSAA to VLVVV, abrogated cleavage by EhROM1. Note that while the amount of AMA1 in media was comparable with TgROM5, wild-type AMA1 was cleaved much better by TgROM5 than its TM mutant form, as assessed by the low level of AMA1 precursor detected in cells (arrow). (C) The relative preference of EhROM1 for the TM segments of BAEBL and AMA1 were compared by assessing cleavage of BAEBL ("B" label above Western, black color in diagram) versus BAEBL harboring the TM of AMA1 ("BA" label above Western, white segment in diagram). EhROM1 cleaved the TM of BAEBL more efficiently than that of AMA1 (arrow in top panel), which is similar to PfROM4, but unlike PfROM1. Note that much of the PfROM1-cleaved product was retained in cells and not secreted (arrow in bottom panel). (D) Cleavage of AMA1 ("A" label above Western, white color in diagram) versus AMA1 containing the TM of BAEBL (" $\mathrm{AB}^{\prime}$ label above Western, black segment in diagram) were compared. While lower levels of EhROM1 resulted in almost no AMA1 cleavage, it efficiently cleaved AMA1 containing the TM of BAEBL under identical conditions. In all cases, media and cell fractions were analyzed by anti-GFP Western, with prestained mass standards shown to the left of each image. mode of substrate specificity reminiscent of PfROM4. To explore this further, we compared the ability of EhROM1 to cleave BAEBL and BAEBL harboring the transmembrane segment from AMA1, a Plasmodium adhesin with some Spitz-like substrate character that required high levels of EhROM1 for cleavage (Figs. 2C, 3B). We previously determined that Plasmodium PfROM1 and PfROM4 have reciprocal specificity against this pair of substrates (Baker et al. 2006): Swapping the transmembrane domains of BAEBL and AMA1 resulted in a swap of relative substrate preference by PfROMs 1 and 4 (Fig. 3C). Under these conditions, EhROM1 displayed potent activity against BAEBL, but cleaved BAEBL harboring the AMA1 transmembrane domain much less efficiently. Note that both substrates were cleaved efficiently by TgROM5, a rhomboid protease from Toxoplasma gondii that displays "dual" specificity and cleaves both Spitz and parasite adhesins.

We also examined cleavage of the reciprocal chimera in which the transmembrane segment of BAEBL was introduced into AMA1. While AMA1 was cleaved inefficiently when EhROM1 was expressed at lower levels, placing just the transmembrane segment of BAEBL into AMA1 dramatically increased its cleavage by EhROM1 (Fig. 3D). Note that TgROM5 cleaved both molecules efficiently (Fig. 3D), consistent with the notion that it's a "dual specificity" protease. Collectively, these chimera-mapping experiments indicate that EhROM1 is specialized for cleavage of a distinct, non-Spitz-like class of substrates. This alternate specificity is analogous to the atypical mode of specificity observed in PfROM4, but different from the specificity of all nonprotozoan rhomboid enzymes studied thus far.

\section{Localization of EhROM1 in E. histolytica trophozoites}

Having discovered that EhROM1 is an active intramembrane serine protease with atypical substrate specificity, we next examined its subcellular localization in E. histolytica trophozoites in order begin addressing its cellular function. To this end, we sought to examine the endogenous protein, rather than an epitope-tagged and overexpressed transgene, in order to avoid potential misleading artifacts. We therefore generated a polyclonal antibody to the first 89 amino acids comprising its cytoplasmic $\mathrm{N}$ terminus. Specificity was confirmed by detection of EhROM1 in transfected COS cells, with little or no immunoreactivity with untransfected or PfROM1transfected COS cell lysates (Fig. 4A). Western analysis of ameba lysates identified a single band around $36 \mathrm{kDa}$, the expected size for EhROM1 (Fig. 4B).

Immunolocalization of EhROM1 under axenic conditions revealed it resides both on the cell surface and in internal punctate structures, as well as occasionally in the membrane of some internal vesicles (Fig. 4C). To define further these internal structures, we costained trophozoites with antibodies to the heavy subunit of the Gal/GalNAc lectin (Hgl), which labels surface and interior vesicles, Amoebapore-A (Ap-A), which labels acidic compartments/lysosomes, and Rab7a, which stains 
Baxt et al.

Figure 4. Localization of endogenous EhROM1 in E. histolytica trophozoites. (A) Specificity of the anti-EhROM1 antisera was evaluated using transfected COS cells. Total lysates from HA-PfROM1-transfected (PfR1), untransfected (-), or HA-EhROM1-transfected (EhR1) COS cells were probed with anti-HA (green) and anti-EhROM1 (red), and were detected with infrared dye-conjugated secondary antibodies (merged image is shown). The anti-HA signal detected both transfected HA-PfROM1 and HA-EhROM1 proteins. Note that the anti-EhROM1 antiserum detected the HA-tagged EhROM1 in transfected cells (as well as some degradation products), but not cellular protein in untransfected cells or PfROM1. (B) Anti-EhROM1 polyclonal antiserum was used to probe an ameba trophozoite lysate. A single band $~ 36$ $\mathrm{kDa}$ was visible, indicating specificity for a single protein of the predicted size for EhROM1. (C) Localization of EhROM1 was determined using indirect immunofluorescence on trophozoites for EhROM1 and a number of other markers including $\mathrm{Hgl}$, Rab7a, and Ap-A. EhROM1 was located on the surface of the parasite overlapping with $\mathrm{Hgl}$ (arrows), as well as in interior vesicles and punctate areas, some of which overlapped with Rab7a and Ap-A (arrows). (D) Parasites were cocultured without (-) or with (+) sRBCs for $15 \mathrm{~min}$ and stained with anti-EhROM1 and anti-Hgl. Note that an increase in interior vesicles stained by both anti-EhROM1 and anti-Hgl (arrows) occurred under phagocytic conditions.

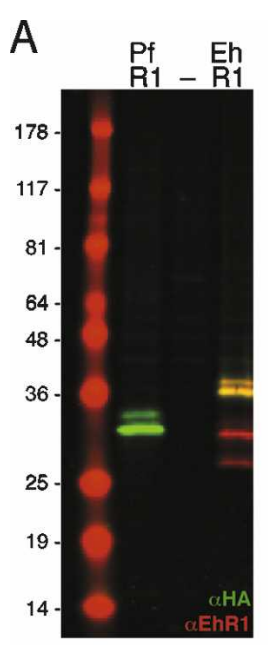

B

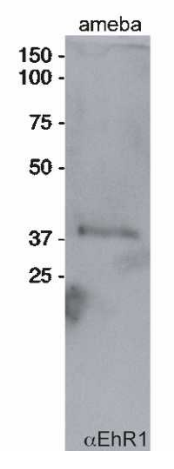

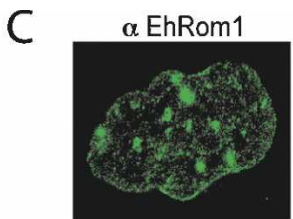
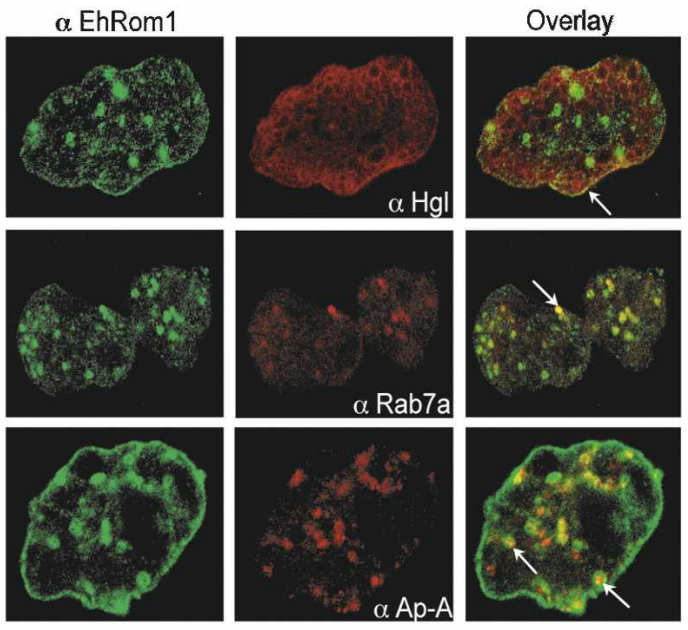

D
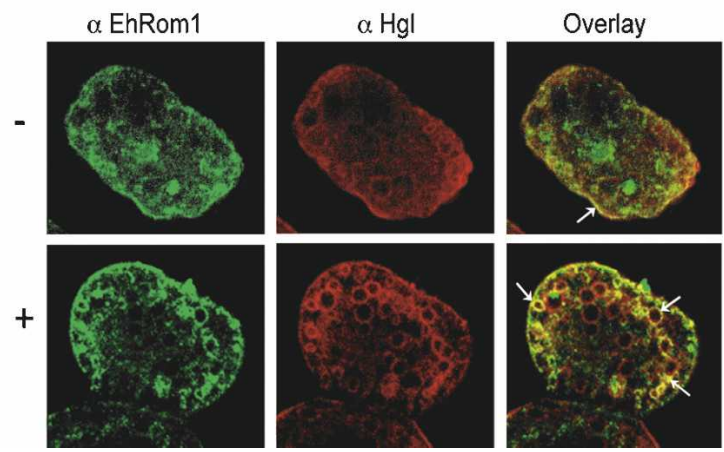

prephagosomal vesicles (Petri et al. 1990; Andra et al. 2003; Saito-Nakano et al. 2004). We discovered that the surface staining of EhROM1 and Hgl overlapped; however, the internal vesicles containing $\mathrm{Hgl}$ did not overlap with internal structures marked by EhROM1 (Fig. 4C). Some punctate structures containing EhROMl overlapped with Ap-A and Rab7a, suggesting some lysosomal and some prephagosomal localization, respectively (Fig. 4C).

E. histolytica is a professionally phagocytic cell that ingests bacteria, red blood cells, and cellular debris, and phagocytosis is believed to contribute to virulence (Orozco et al. 1983; Voigt and Guillen 1999). Interestingly, EhROM1 localization changed during erythrophagocytosis to internal vesicles as early as $10-15 \mathrm{~min}$ after incubation with red blood cells (Fig. 4D). Some of these vesicles contained ingested red blood cells, but the majority lacked obviously ingested particles. While EhROM1 did not colocalize with the Gal/GalNAc lectin $\mathrm{Hgl}$ in vesicles under resting conditions (only on the surface), during phagocytosis the redistributed EhROM1 now colocalized with $\mathrm{Hgl}$ in interior vesicles, suggesting that it may be actively transported to new vesicle subsets in response to phagocytosis (Fig. 4D).
Candidate substrate search identifies a family of $\mathrm{E}$. histolytica surface lectins

Having examined the substrate specificity characteristics of EhROM1 and discovered it to be relocalized to phagocytic vesicles upon feeding, we performed a candidate substrate search in order to begin addressing its cellular role. We examined annotated genes for rhomboid substrate sequences, focusing on proteins identified to be surface membrane proteins or in phagosomes (Okada et al. 2005). This analysis identified the conserved family of $\mathrm{Gal} / \mathrm{GalNAc}$ lectin heavy-chain subunits (i.e., $\mathrm{Hgl}$ ) encoded by EH1_012270, EHI_077500, EHI_133900, and EHI_042370, as well as lectin EHI_044650, as putative substrates. The functional lectins are composed of a transmembrane-anchored heavy subunit $(\mathrm{Hgl})$, which is linked via disulfide bonds to a GPI-anchored light subunit (Lgl) (for review, see Frederick and Petri 2005). The heavy subunit of the lectin has crucial roles in multiple aspects of amebic pathogenesis, including invasion (adherence, cytolysis, induction of apoptosis, and signaling), immune evasion (complement resistance and surface receptor shedding), and development (stage conversion during encystation) (for review, see Petri et al. 2002). 
Stretches of alanine and glycine residues in the upper region of $\mathrm{Hgl}$ transmembrane segments made them promising candidates for EhROM1 substrates (Fig. 5A). Note that this family includes the $\mathrm{Hgl}$ lectin heavy subunit that relocalized to vesicles with EhROM1 during phagocytosis (Fig. 4D).

To assess whether ameba lectins could be cleaved by EhROM1, we cloned them into a mammalian expression vector after an $\mathrm{N}$-terminal GFP tag. Due to their large ectodomains with poorly defined domain structure, we cloned at least two different $\mathrm{N}$-terminal truncations of each of EH1_012270, EHI_077500, EHI_133900, EHI_042370, and EHI_044650. Although we were able to achieve robust expression of all constructs, all but the longest version of EHI_044650 were localized to the ER of COS cells, implying that their ectodomains were unfolded and thus retained in the ER as a result of quality control (data not shown). This may be due to misfolding of cysteine-rich regions present in the truncated ectodomains. We therefore focused on the EHI_044650 con-

A

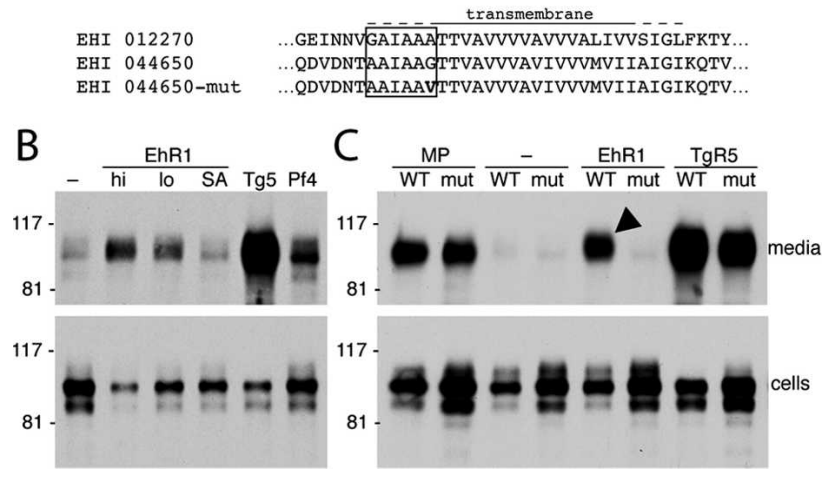

Figure 5. Ameba lectins are substrates for EhROM1. (A) Transmembrane sequences (overlined) of ameba lectins EHI_012270 (Hgl) and EHI_044650 contain small residues in their upper regions (boxed) that are hallmarks of rhomboid substrates. The sequence of a glycine-to-valine mutant of EHI_044650 is also shown for comparison (mut). Only TM and surrounding residues are shown; the N-terminal lectin ectodomains are to the left, and cytoplasmic tails are to the right. (B) GFP-tagged $E$. histolytica heavy-chain lectin (EHI_044650) construct was cotransfected into COS cells with either high or low amounts of EhROM1 (hi or lo, respectively), the EhROM1 serine-to-alanine mutant (SA), or TgROM5 and PfROM4. Media and cell fractions were analyzed by anti-GFP Western, with prestained molecular mass standards denoted to the left of each image. EhROM1, TgROM5, and PfROM4 were able to process the GFP-lectin, as assessed by accumulation of the cleaved form in cell culture media. $(C)$ Cleavage of wild type (WT) versus a glycine-to-valine mutant (mut) EHI_044650 lectin by cellular metalloproteases (MP), EhROM1 (EhR1, wild-type product denoted by arrow), and TgROM5 (TgR5) were compared. Note that the glycine-to-valine mutant abolished cleavage by EhROM1 but not by endogenous cellular metalloproteases (MP). Cells transfected with only the GFP-lectin construct were incubated without metalloprotease inhibitors (MP) to allow processing by cellular metalloproteases (inhibitors were included in the remaining transfections to facilitate detection of rhomboid-cleaved products). struct that contained the C-terminal third (residues 809 to 1207$)$ of the lectin.

EHI_044650 was cleaved in a dose-dependent manner by EhROM1 when they were cotransfected into COS cells, with high amounts of enzyme producing more cleaved product released into cell culture media than the lower level of EhROM1 (Fig. 5B). The serine mutant of EhROM1 failed to display any activity above background against the lectin construct. Moreover, rhomboid enzymes from other parasites were able to cleave the lectin to different degrees: TgROM5 cleaved the lectin with very high efficiency, while PfROM4 displayed low activity.

To assess whether the amebic lectin is a specific substrate for EhROM1, we mutated the lectin's transmembrane sequence. Rhomboid enzymes typically require helix-breaking residues within the upper register of their substrate transmembrane segments, and the ameba lectin contains an alanine-rich region followed by a glycine (Fig. 5A). Mutation of this glycine to valine in the lectin abolished cleavage by EhROM1, while reducing its cleavage by TgROM5 (Fig. 5C). Both the wild-type and valine mutant lectins were shed from COS cells at equivalent levels by endogenous, cell-encoded surface metalloproteases. This observation further suggested that the block in processing was specific to EhROM1 cleavage, since both lectins were expressed, trafficked to the cell surface, and cleaved by juxtamembrane proteases at similar levels. Thus, ameba surface lectins may be specific and physiological substrates for EhROM1 under a variety of conditions including phagocytosis.

\section{EhROM1 dramatically relocalizes to the base of the uroid during capping}

Ameba lectins are important for cell adhesion, but are also known to be shed from the cell surface to evade the host immune system. E. histolytica employs a number of strategies for immune evasion, the most unique of which is surface receptor capping, whereby surface receptors susceptible to host immune components are polarized to the posterior end of the cell (or uroid) and released into the culture medium (Calderon 1980; Espinosa-Cantellano and Martinez-Palomo 1994). The Gal/ GalNAc lectin has been shown to relocalize to caps (Marion et al. 2004), and since we identified a heavy subunit of an amebic lectin as a substrate for EhROM1, we investigated whether EhROM1 would change localization in response to capping. We induced cap formation using Concanavalin A (Arhets et al. 1995) and observed the expected relocalization of $\mathrm{Hgl}$ to one end of the parasite into caps (Fig. 6). Interestingly, we discovered a dramatic localization of EhROM1 to the base of the formed caps. In order to ascertain the possible role of EhROM1 in more detail, we examined multiple parasites at different stages of the capping process (Espinosa-Cantellano and Martinez-Palomo 1994). EhROM1 surrounded the cap when it was beginning to form (Fig. 6, left panel), localized to a cup-like structure around the base of the fully formed cap (Fig. 6, middle panel), and appeared to 
Baxt et al.

A

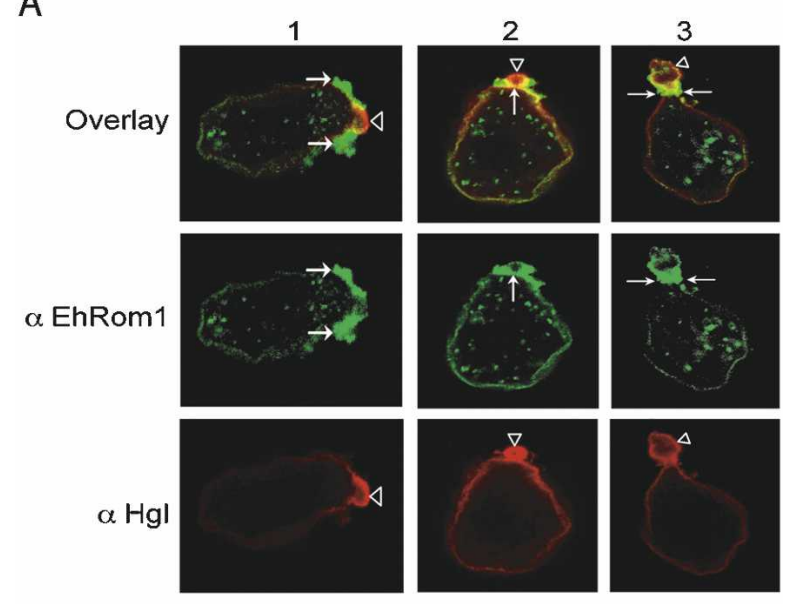

B

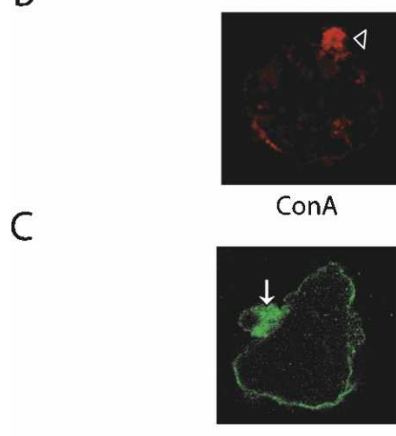

$\alpha$ EhROM1

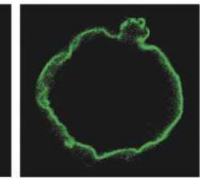

$\alpha$ SREHP

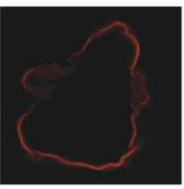

$\alpha$ SREHP

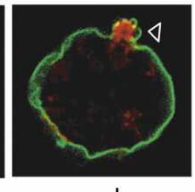

overlay

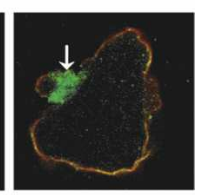

overlay

Figure 6. EhROM1 relocalizes to the uroid during capping. (A) Capping was induced in trophozoites followed by staining for $\mathrm{Hgl}$ and EhROM1. A dramatic relocalization of $\mathrm{Hgl}$ to the cap at the posterior end of the parasite was observed. EhROM1 also showed a dramatic localization to the base of the cap but not in the cap itself. Three representative parasites are shown at each stage $(1,2$, and 3$)$ with arrows indicating EhROM1 localization and arrowheads indicating caps marked with Hgl. (B) SREHP did not relocalize to the cap during uroid formation. The uroid is marked by Concanavalin A (arrowhead), while SREHP remains on the surface of the entire parasite, rather than moving specifically to the cap. $(C)$ EhROM1 localizes to the base of the cap (arrows), whereas SREHP did not enrich around the base of the cap.

surround the neck of the cap when it was pinching off for release (Fig. 6, right panel). A moderate amount of EhROM1 also remained on the surface as well as in interior vesicles and punctate structures during capping.

The localization of EhROM1 around the cap structure base was unusual. To examine whether it was specific, we tested a third unrelated protein, the serine-rich $E$. histolytica protein (SREHP), a highly abundant and antigenic surface protein (Teixeira and Huston 2008). Under capping conditions, SREHP remained abundant on the surface of the entire parasite, with little enrichment in the cap area (Fig. 6B). In costaining experiments with EhROM1, SREHP was present around the entire parasite while EhROM1 formed a concentrated cuplike structure at the base of the cap (Fig. 6C). EhROM1 is the first pro-

tein to be identified specifically at the base of the cap rather than in the cap itself, raising the exciting possibility that it plays a specific role in late steps of the capping process and/or cap shedding during immune evasion.

\section{Discussion}

Rhomboid enzymes are unusual among regulatory factors in being conserved across both multicellular and unicellular organisms, including bacteria and protozoa (Koonin et al. 2003; Dowse and Soldati 2005). Recent work has implicated these proteases in adhesin cleavage during invasion of host cells by obligate intracellular parasites of the phylum Apicomplexa, but little is known about rhomboid function in other pathogens (Brossier et al. 2005; Dowse et al. 2005; Baker et al. 2006; O'Donnell et al. 2006; Trasarti et al. 2007). We investigated the only rhomboid enzyme encoded by the extracellular pathogen E. histolytica. EhROM1 was an active intramembrane protease, but displayed the atypical substrate specificity analogous to PfROM4. EhROM1 was localized to the parasite surface and internal structures, but relocalized to vesicles during phagocytosis and to the uroid during capping. We discovered that a parasite lectin is a substrate for EhROM1, and EhROM1 colocalized with a parasite transmembrane lectin during phagocytosis and capping. These observations collectively suggest that EhROM1 functions to cleave parasite surface lectins under various conditions. However, we emphasize that further analysis directly in ameba cells is required to verify both that lectins are physiological targets of EhROM1 and the relative importance of this cleavage event.

The amebic Gal/GalNAc lectin is one of the most abundant proteins in the parasite and is known to be released into the culture medium at levels comparable to those remaining in the parasite (Petri et al. 1990). EhROM1 is the first identified sheddase for $\mathrm{Hgl}$, and both colocalize on the parasite surface under resting conditions; thus a basal level of surface shedding of $\mathrm{Hgl}$ by EhROM1 may be occurring at all times. A more informative observation may be the regulated redeployment of EhROM1 to the base of the uroid during capping. Effective mechanisms of immune evasion are imperative for E. histolytica to survive as an extracellular pathogen, and surface receptor capping is an important strategy (Calderon 1980; Espinosa-Cantellano and MartinezPalomo 1994). Intriguingly, EhROM1 was localized to the base of the cap rather than throughout its surface, implying that it is not the cargo, but rather may be a factor that contributes to cap formation or release. It seems plausible that a sheddase activity provided by EhROM1 may be important for these events, either by releasing individual lectins from the surface or in facilitating release of the cap structure as a whole. The redistribution of EhROM1 to vesicles during phagocytosis could also suggest that lectin release is important during phagocytosis, perhaps to release cargo if lectins act as surface receptors in this process. However, both the processes of capping and phagocytosis, as well as the role of 
lectins themselves, are relatively poorly defined. While genetic approaches would be invaluable in delineating these processes further, knockouts are not available in $E$. histolytica, and current knockdown strategies have proven difficult with EhROM1 despite repeated attempts (data not shown), perhaps because it is among the most highly active rhomboid enzymes analyzed. Nevertheless, this is the first implication of any rhomboid enzyme in immune evasion.

Although the function of rhomboid enzymes in remarkably diverse pathogens has previously been puzzling, our observations now imply that a common feature of rhomboid enzymes might be to shed adhesive surface proteins. For Apicomplexan parasites, rhomboid activity breaks down the moving junction at the end of host cell invasion, while in ameba rhomboid activity would cleave lectins during receptor capping for immune evasion. Thus, although the consequence of EhROM1 catalysis is remarkably different in ameba versus other systems, our results now suggest broad and intriguing parallels.

Change of subcellular localization is also known to be a major route for regulating rhomboid proteolytic activity in widely diverse processes. During Drosophila development, Spitz must be relocalized from the ER to the Golgi apparatus, where Rhomboid-1 resides, by the transport function of Star to initiate Spitz activation (Lee et al. 2001; Tsruya et al. 2002). Similarly, Toxoplasma and Plasmodium transmembrane adhesins are held in internal microneme organelles prior to invasion, while PfROM4 and TgROM5 reside on the parasite surface (Brossier et al. 2005; O'Donnell et al. 2006). Only upon secretion of transmembrane adhesins to the cell surface and translocation of the adhesin:host receptor complexes to the posterior of the parasite do substrate and rhomboid protease meet, ensuring that disengagement of junctions does not occur prematurely. Relocalization of EhROM1 to vesicles triggered by phagocytosis and the cap triggered by immune attack is analogous to these events and implies that EhROM1 proteolytic activity and therefore function is rapidly and tightly regulated in response to specific stimuli, despite its gene being broadly expressed. Interestingly, this relocalization event represents a rhomboid protease, rather than a substrate, changing its subcellular localization (Urban 2006). Recently, the protease PfROM1 itself was found to be secreted from internal organelles to the surface during Plasmodium invasion (Singh et al. 2007).

Another notable parallel is the substrate specificity of EhROM1. At the biochemical level, EhROM1 displayed the atypical mode of substrate specificity analogous to the PfROM4/TgROM5 subclass of Plasmodium and Toxoplasma rhomboid enzymes involved in invasion, but unlike those of other rhomboid enzymes from Plasmodium or Toxoplasma (Brossier et al. 2005; Baker et al. 2006) or those from bacteria, plants, or animals (Urban et al. 2002b; Urban and Freeman 2003; Kanaoka et al. 2005). This is the first description of this new mode of substrate specificity beyond Apicomplexa and raises the possibility that it may mark a more broad evolutionary turn in the cellular and biochemical function of rhomboid enzymes. On a practical level, multiple substrates might be expected to exist for such a membrane protein sheddase, and further work to delineate the molecular basis of this atypical specificity may facilitate identification of other potential EhROM1 substrates.

Finally, since E. histolytica infection is a major health problem globally yet this pathogen is not amenable to genetic dissection, a practical implication of our work is the identification of a possible therapeutic target for future consideration. EhROM1 is the only protease known to cleave ameba lectins, which are widely known to play key roles in many ameba processes that contribute to virulence (for review, see Petri et al. 2002). The shared biochemical properties with Apicomplexan rhomboid enzymes is another serendipitous advantage in developing EhROM1 into a therapeutic target. Moreover, the presence of a single E. histolytica rhomboid suggests that functional redundancy is unlikely to be encountered in targeting EhROM1. These observations now provide unanticipated and potentially important avenues of research that may have practical applications.

\section{Materials and methods}

\section{Identification of putative E. histolytica rhomboid proteases}

Putative rhomboid proteases were identified in the E. histolytica genome sequence using the Blocks cobbler sequence for Rhomboid protease (IPB002610) (http://blocks.fhcrc.org/blocksbin/getblock.sh?IPB002610). This entry contains the amino acid sequence corresponding to the most highly conserved regions across all known rhomboid proteases across multiple alignments. A BlastP of the cobbler sequence against the $E$. histolytica genome (http://tigrblast.tigr.org/er-blast/index.cgi?project= eha1) resulted in 10 potential matches. To determine which of these represented potential rhomboid proteases, protein sequences were analyzed using the CDART tool for identifying conserved protein domains (http://www.ncbi.nlm.nih.gov/ Structure/lexington/lexington.cgi?cmd=rps). Results were filtered to show hits with a probability value $>0.01$ and rhomboid protease domains were identified in five genes, accession numbers EAL46300.1, EAL44183.1, EAL47860.1, EAL49376.1, and EAL51684.1. Identification of homologs in Entamoeba dispar and Entamoeba invadens was performed using tBlastX against the available sequence for each genome (http://www.ncbi.nlm. nih.gov/sutils/blast_table.cgi?taxid=Protozoa), and the highest match for each was noted.

\section{EhROM1 expression and cleavage in COS cells}

Rhomboid enzyme activity was assayed in transiently transfected mammalian cells as described in detail previously (Baker et al. 2006). Expression of all DNA constructs was driven by the CMV promoter of the pcDNA3.1 vector (Invitrogen). Endogenous genomic and synthetic, codon-optimized EhROM1 (synthesized by GeneArt) were tagged at their extreme $\mathrm{N}$ termini with a triple hemagglutinin tag. All adhesins, their corresponding mutants, and Spitz were tagged at their $\mathrm{N}$ termini with a GFP tag, as described previously (Baker et al. 2006). Mutagenesis was accomplished using the Quik-Change method (Stratagene) and verified by sequencing.

COS cells were maintained in Dulbecco's Modified Eagle's 
Medium (DMEM) supplemented with L-glutamine and 10\% fetal calf serum. For transfection, cells were seeded in six-well plates and transfected with 250 ng of GFP-adhesin or GFP-Spitz pcDNA3.1 constructs (and Star to facilitate Spitz transport) with each pcDNA3.1-ROM construct. The final amount of DNA in each transfection was adjusted to $1 \mu \mathrm{g}$ with pBluescript, and transfection complexes were formed using FuGene6 (Roche) according to the manufacturer's instructions. Cells were washed $18 \mathrm{~h}$ after transfection, and serum-free DMEM was conditioned for the following $24 \mathrm{~h}$. Metalloprotease inhibitors were included in the media to reduce background ectodomain shedding of the GFP-tagged substrates.

Media and cell lysate samples were resolved by SDS-PAGE under denaturing conditions and probed with anti-GFP or antiHA antibodies (Santa Cruz Biotechnologies). The resulting immune complexes were revealed with enhanced chemiluminescence (Amersham), detected with X-ray film, and digitized on an Umax scanner in transmissive mode, operating through Photoshop software (Adobe). For two-color detection, anti-HA and anti-EhROM1 complexes were detected using anti-rabbit IRDye-800 (Rockland) and anti-rat Alexa Fluor-680 (Invitrogen) secondary antibodies, respectively, and imaged using a LiCor Odyssey infrared scanner.

\section{Entamoeba histolytica lectin substrate constructs}

Lectin heavy subunits were amplified from $E$. histolytica genomic DNA and cloned after GFP that had been inserted into pcDNA3.1 (Invitrogen). Since the domain structure of E. histolytica lectins has not been well delineated, the $\mathrm{N}$-terminal start sites were chosen to lie between regions that align as putative domains with other unrelated proteins. Cells were cotransfected with EhROM1 using FuGene6, and media and cell samples were examined by anti-GFP Western analysis as described above.

\section{Antibody production}

Polyclonal anti-EhROM1 antibodies were produced by expressing the first 89 amino acids of EhROM1, comprising the $\mathrm{N}$ terminal cytoplasmic domain of the protein, as a GST fusion protein in the bacterial expression vector (pGEX-6P-1). Protein production was accomplished as described (Urban and Wolfe 2005 ) by adding $250 \mu \mathrm{M}$ IPTG and incubating for $3 \mathrm{~h}$ at $27^{\circ} \mathrm{C}$. The GST fusion protein was isolated according to standard protocols and sent to Harlan Bioproducts for Science (http:// www.hbps.com). Antibody production was carried out according to standard rat injection protocol (http://www.hbps.com/ index.htm).

\section{E. histolytica culture conditions and indirect immunofluorescence assays}

E. histolytica (HM-1:IMSS) was grown axenically in TYI-S-33 medium under standard culture conditions at $36.5^{\circ} \mathrm{C}$ (MacFarlane and Singh 2006). Log-phase parasites grown in culture tubes were put for $10 \mathrm{~min}$ at $4^{\circ} \mathrm{C}$ to release parasites, placed in chamber slides, and allowed to adhere for $30 \mathrm{~min}$ at $37^{\circ} \mathrm{C}$. Amebas were rinsed twice with room temperature PBS, fixed in $4 \%$ ultrapure, methanol-free formaldehyde (Polysciences) for 10 min, followed by permeabilization in $100 \% \mathrm{EtOH}$ for $20 \mathrm{~min}$ at room temperature. The reaction was blocked with $3 \%$ BSA-PBS for $30 \mathrm{~min}$, followed by staining for $1 \mathrm{~h}$ with primary antibody diluted in $1 \%$ BSA-PBS. Primary antibody dilutions used for these experiments were anti-EhROM1 (1:20), anti heavy lectin 3F4 (1:50) (kind gift of W.A. Petri, Jr.), anti-Rab7A (1:100) (kind gift of Tomo Nozaki), anti-amebapore A (1: 500) (kind gift of Matthias Leippe), and anti SREHP 1:100 (kind gift of Christopher Huston). Parasites were rinsed twice with $1 \times$ PBS and incubated with appropriate secondary antibodies (Alexa 488 anti-mouse 1:1000, Alexa-488 anti-rabbit 1:1000, Alexa 594 anti-mouse 1:500, Alexa 488 anti-rat 1:1000, streptavidin Texas Red 1:500) (Molecular Probes, Vector Laboratories) for $1 \mathrm{~h}$ in the dark. After staining, amebas were rinsed twice with PBS, mounted on chamber slides with vectashield solution, and imaged with a BioRad confocal and Nikon Eclipse TE300 microscope using the Laser Sharp 2000 program. A single 0.5- $\mu$ m slice is shown for each figure.

\section{Phagocytosis assays}

Phagocytosis assays were performed using sheep red blood cells (sRBCs) (Hemostat Co.). For all assays parasites were seeded in chamber slides and allowed to adhere for $30 \mathrm{~min}$ at $37^{\circ} \mathrm{C}$ and incubated with $\sim 1 \times 10^{5} \mathrm{sRBCs}$ per milliliter for $15 \mathrm{~min}$, rinsed twice with PBS, fixed, and stained as described above.

\section{Induction of capping in trophozoites}

Capping was induced in trophozoites as described previously (Arhets et al. 1995). Trophozoites were chilled and transferred to chamber slides where they were allowed to adhere for $30 \mathrm{~min}$ at $37^{\circ} \mathrm{C}$. Parasites were rinsed twice with ice cold PBS and incubated with PBS containing $20 \mu \mathrm{g} / \mathrm{mL}$ biotinylated Con A /Vector Laboratories, Sigma) for $1 \mathrm{~h}$ on ice. Parasites were then incubated for $15 \mathrm{~min}$ at $37^{\circ} \mathrm{C}$ to induce cap formation. Following cap formation parasites were rinsed with PBS, fixed, and stained as described above.

\section{Acknowledgments}

We are grateful to all members of the Singh and Urban labs for helpful advice and input. L.B. acknowledges Manuel Amieva for help with microscopy and Shumin Tan for initial help with cloning EhROM1. We thank W.A. Petri, Jr., Tomo Nozaki, Matthias Leippe, and Christopher Huston for gifts of the anti-Hgl, anti-Rab7A, anti-amebapore A, and anti-SREHP antibodies, respectively. S.U.. is grateful to Chelsea Newhouse for administrative assistance. This work was supported by NIH grant R01AI066025 (to S.U.), a career award from the Burroughs-Wellcome Fund (to S.U.), a David and Lucile Packard Foundation Award for Science and Engineering (to S.U.), an NIH training grant T32-AI07328 (to L.B.), and NIH grant AI-053724 (to U.S.).

\section{References}

Ackers, J., Clark, C.G., Diamond, L.S., Duchêne, M., Espinosa Cantellano, M., Jackson, T.F.H.G., Martínez-Palomo, A., Mirelman, D., Muñoz Hernández, O. et al. 1997. WHO/ PAHO/UNESCO report. A consultation with experts on amoebiasis. Mexico City, Mexico 28-29 January, 1997. Epidemiol. Bull. PAHO 18: 13-14.

Andra, J., Herbst, R., and Leippe, M. 2003. Amoebapores, archaic effector peptides of protozoan origin, are discharged into phagosomes and kill bacteria by permeabilizing their membranes. Dev. Comp. Immunol. 27: 291-304.

Arhets, P., Gounon, P., Sansonetti, P., and Guillen, N. 1995. Myosin II is involved in capping and uroid formation in the human pathogen Entamoeba histolytica. Infect. Immun. 63: $4358-4367$.

Baker, R.P., Wijetilaka, R., and Urban, S. 2006. Two plasmo- 
dium rhomboid proteases preferentially cleave different adhesins implicated in all invasive stages of malaria. PLoS Pathog. 2: e113. doi: 10.1371/journal.ppat.0020113.

Baker, R.P., Young, K., Feng, L., Shi, Y., and Urban, S. 2007. Enzymatic analysis of a rhomboid intramembrane protease implicates transmembrane helix 5 as the lateral substrate gate. Proc. Nat1. Acad. Sci. 104: 8257-8262.

Ben-Shem, A., Fass, D., and Bibi, E. 2007. Structural basis for intramembrane proteolysis by rhomboid serine proteases. Proc. Natl. Acad. Sci. 104: 462-466.

Bier, E., Jan, L.Y., and Jan, Y.N. 1990. rhomboid, a gene required for dorsoventral axis establishment and peripheral nervous system development in Drosophila melanogaster. Genes \& Dev. 4: 190-203.

Brossier, F., Jewett, T.J., Sibley, L.D., and Urban, S. 2005. A spatially localized rhomboid protease cleaves cell surface adhesins essential for invasion by Toxoplasma. Proc. Natl. Acad. Sci. 102: 4146-4151.

Calderon, J. 1980. Dynamic changes on the surface of Entamoeba induced by antibodies. Arch. Invest. Med. (Mex.) 11 (Suppl. 1): 55-61.

Carruthers, V.B., Sherman, G.D., and Sibley, L.D. 2000. The Toxoplasma adhesive protein MIC2 is proteolytically processed at multiple sites by two parasite-derived proteases. $J$. Biol. Chem. 275: 14346-14353.

Cipolat, S., Rudka, T., Hartmann, D., Costa, V., Serneels, L., Craessaerts, K., Metzger, K., Frezza, C., Annaert, W., D'Adamio, L., et al. 2006. Mitochondrial rhomboid PARL regulates cytochrome $\mathrm{c}$ release during apoptosis via OPA1dependent cristae remodeling. Cell 126: 163-175.

Coudrier, E., Amblard, F., Zimmer, C., Roux, P., Olivo-Marin, J.C., Rigothier, M.C., and Guillen, N. 2005. Myosin II and the Gal-GalNAc lectin play a crucial role in tissue invasion by Entamoeba histolytica. Cell. Microbiol. 7: 19-27.

Dowse, T.J. and Soldati, D. 2005. Rhomboid-like proteins in Apicomplexa: Phylogeny and nomenclature. Trends Parasitol. 21: 254-258.

Dowse, T.J., Pascall, J.C., Brown, K.D., and Soldati, D. 2005. Apicomplexan rhomboids have a potential role in microneme protein cleavage during host cell invasion. Int. J. Parasitol. 35: 747-756.

Ehrenkaufer, G.M., Haque, R., Hackney, J.A., Eichinger, D.J., and Singh, U. 2007. Identification of developmentally regulated genes in Entamoeba histolytica: Insights into mechanisms of stage conversion in a protozoan parasite. Cell. Microbiol. 9: 1426-1444.

Espinosa-Cantellano, M. and Martinez-Palomo, A. 1994. Entamoeba histolytica: Mechanism of surface receptor capping. Exp. Parasitol. 79: 424-435.

Frederick, J.R. and Petri Jr., W.A. 2005. Roles for the galactose-/ $\mathrm{N}$-acetylgalactosamine-binding lectin of Entamoeba in parasite virulence and differentiation. Glycobiology 15: 53R59R.

Herlan, M., Vogel, F., Bornhovd, C., Neupert, W., and Reichert, A.S. 2003. Processing of Mgm1 by the rhomboid-type protease Pcpl is required for maintenance of mitochondrial morphology and of mitochondrial DNA. J. Biol. Chem. 278: 27781-27788.

Howell, S.A., Hackett, F., Jongco, A.M., Withers-Martinez, C., Kim, K., Carruthers, V.B., and Blackman, M.J. 2005. Distinct mechanisms govern proteolytic shedding of a key invasion protein in apicomplexan pathogens. Mol. Microbiol. 57: 1342-1356.

Jeyaraju, D.V., Xu, L., Letellier, M.C., Bandaru, S., Zunino, R., Berg, E.A., McBride, H.M., and Pellegrini, L. 2006. Phosphorylation and cleavage of presenilin-associated rhomboid-like protein (PARL) promotes changes in mitochondrial morphology. Proc. Natl. Acad. Sci. 103: 18562-18567.

Kanaoka, M.M., Urban, S., Freeman, M., and Okada, K. 2005. An Arabidopsis Rhomboid homolog is an intramembrane protease in plants. FEBS Lett. 579: 5723-5728.

Koonin, E.V., Makarova, K.S., Rogozin, I.B., Davidovic, L., Letellier, M.C., and Pellegrini, L. 2003. The rhomboids: A nearly ubiquitous family of intramembrane serine proteases that probably evolved by multiple ancient horizontal gene transfers. Genome Biol. 4: R19. doi: 10.1186/gb-2003-4-3r19.

Lee, J.R., Urban, S., Garvey, C.F., and Freeman, M. 2001. Regulated intracellular ligand transport and proteolysis control EGF signal activation in Drosophila. Cell 107: 161-171.

Lemberg, M.K. and Freeman, M. 2007. Functional and evolutionary implications of enhanced genomic analysis of rhomboid intramembrane proteases. Genome Res. 17: 1634-1646.

Lemieux, M.J., Fischer, S.J., Cherney, M.M., Bateman, K.S., and James, M.N. 2007. The crystal structure of the rhomboid peptidase from Haemophilus influenzae provides insight into intramembrane proteolysis. Proc. Natl. Acad. Sci. 104: 750-754.

Loftus, B., Anderson, I., Davies, R., Alsmark, U.C., Samuelson, J., Amedeo, P., Roncaglia, P., Berriman, M., Hirt, R.P., Mann, B.J., et al. 2005. The genome of the protist parasite Entamoeba histolytica. Nature 433: 865-868.

MacFarlane, R.C. and Singh, U. 2006. Identification of differentially expressed genes in virulent and nonvirulent Entamoeba species: Potential implications for amebic pathogenesis. Infect. Immun. 74: 340-351.

Marion, S., Tavares, P., Arhets, P., and Guillen, N. 2004. Signal transduction through the Gal-GalNAc lectin of Entamoeba histolytica involves a spectrin-like protein. Mol. Biochem. Parasitol. 135: 31-38.

McQuibban, G.A., Saurya, S., and Freeman, M. 2003. Mitochondrial membrane remodelling regulated by a conserved rhomboid protease. Nature 423: 537-541.

O'Donnell, R.A., Hackett, F., Howell, S.A., Treeck, M., Struck, N., Krnajski, Z., Withers-Martinez, C., Gilberger, T.W., and Blackman, M.J. 2006. Intramembrane proteolysis mediates shedding of a key adhesin during erythrocyte invasion by the malaria parasite. J. Cell Biol. 174: 1023-1033.

Okada, M., Huston, C.D., Mann, B.J., Petri Jr., W.A., Kita, K., and Nozaki, T. 2005. Proteomic analysis of phagocytosis in the enteric protozoan parasite Entamoeba histolytica. Eukaryot. Cell 4: 827-831.

Opitz, C., Di Cristina, M., Reiss, M., Ruppert, T., Crisanti, A., and Soldati, D. 2002. Intramembrane cleavage of microneme proteins at the surface of the apicomplexan parasite Toxoplasma gondii. EMBO J. 21: 1577-1585.

Orozco, E., Guarneros, G., Martinez-Palomo, A., and Sanchez, T. 1983. Entamoeba histolytica. Phagocytosis as a virulence factor. J. Exp. Med. 158: 1511-1521.

Petri Jr., W.A., Jackson, T.F., Gathiram, V., Kress, K., Saffer, L.D., Snodgrass, T.L., Chapman, M.D., Keren, Z., and Mirelman, D. 1990. Pathogenic and nonpathogenic strains of Entamoeba histolytica can be differentiated by monoclonal antibodies to the galactose-specific adherence lectin. Infect. Immun. 58: 1802-1806.

Petri Jr., W.A., Haque, R., and Mann, B.J. 2002. The bittersweet interface of parasite and host: Lectin-carbohydrate interactions during human invasion by the parasite Entamoeba histolytica. Annu. Rev. Microbiol. 56: 39-64.

Rodriguez, M.A. and Orozco, E. 1986. Isolation and characterization of phagocytosis- and virulence-deficient mutants of Entamoeba histolytica. J. Infect. Dis. 154: 27-32. 


\section{Baxt et al.}

Saito-Nakano, Y., Yasuda, T., Nakada-Tsukui, K., Leippe, M., and Nozaki, T. 2004. Rab5-associated vacuoles play a unique role in phagocytosis of the enteric protozoan parasite Entamoeba histolytica. J. Biol. Chem. 279: 49497-49507.

Singh, S., Plassmeyer, M., Gaur, D., and Miller, L.H. 2007. Mononeme: A new secretory organelle in Plasmodium falciparum merozoites identified by localization of rhomboid-1 protease. Proc. Natl. Acad. Sci. 104: 20043-20048.

Stanley Jr., S.L. 2003. Amoebiasis. Lancet 361: 1025-1034.

Teixeira, J.E. and Huston, C.D. 2008. Participation of the serinerich Entamoeba histolytica Protein in amebic phagocytosis of apoptotic host cells. Infect. Immun. 76: 959-966.

Trasarti, E., Pizzi, E., Pozio, E., and Tosini, F. 2007. The immunological selection of recombinant peptides from Cryptosporidium parvum reveals 14 proteins expressed at the sporozoite stage, 7 of which are conserved in other apicomplexa. Mol. Biochem. Parasitol. 152: 159-169.

Tsruya, R., Schlesinger, A., Reich, A., Gabay, L., Sapir, A., and Shilo, B.Z. 2002. Intracellular trafficking by Star regulates cleavage of the Drosophila EGF receptor ligand Spitz. Genes \& Dev. 16: 222-234.

Urban, S. 2006. Rhomboid proteins: Conserved membrane proteases with divergent biological functions. Genes \& Dev. 20: 3054-3068.

Urban, S. and Freeman, M. 2003. Substrate specificity of rhomboid intramembrane proteases is governed by helix-breaking residues in the substrate transmembrane domain. Mol. Cell 11: 1425-1434.

Urban, S. and Wolfe, M.S. 2005. Reconstitution of intramembrane proteolysis in vitro reveals that pure rhomboid is sufficient for catalysis and specificity. Proc. Natl. Acad. Sci. 102: $1883-1888$.

Urban, S., Lee, J.R., and Freeman, M. 2001. Drosophila rhomboid-1 defines a family of putative intramembrane serine proteases. Cell 107: 173-182.

Urban, S., Lee, J.R., and Freeman, M. 2002a. A family of Rhomboid intramembrane proteases activates all membranetether EGF ligands in Drosophila. EMBO J. 21: 4277-4286.

Urban, S., Schlieper, D., and Freeman, M. 2002b. Conservation of intramembrane proteolytic activity and substrate specificity in eukaryotic and prokaryotic Rhomboids. Curr. Biol. 12: $1507-1512$.

Voigt, H. and Guillen, N. 1999. New insights into the role of the cytoskeleton in phagocytosis of Entamoeba histolytica. Cell. Microbiol. 1: 195-203.

Wang, Y., Zhang, Y., and Ha, Y. 2006. Crystal structure of a rhomboid family intramembrane protease. Nature 444: 179180.

Wu, Z., Yan, N., Feng, L., Oberstein, A., Yan, H., Baker, R.P., Gu, L., Jeffrey, P.D., Urban, S., and Shi, Y. 2006. Structural analysis of a rhomboid family intramembrane protease reveals a gating mechanism for substrate entry. Nat. Struct. Mol. Biol. 13: 1084-1091. 


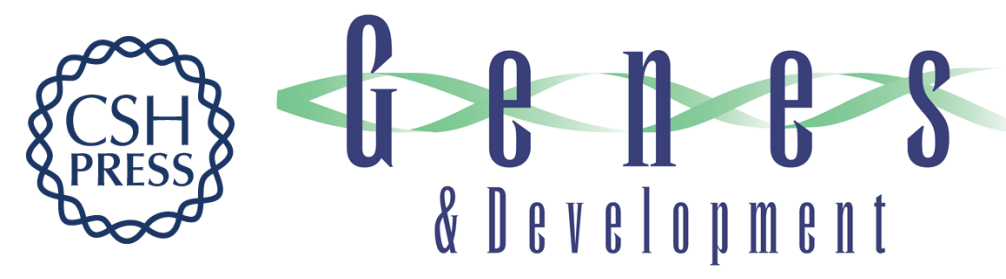

\section{An Entamoeba histolytica rhomboid protease with atypical specificity cleaves a surface lectin involved in phagocytosis and immune evasion}

Leigh A. Baxt, Rosanna P. Baker, Upinder Singh, et al.

Genes Dev. 2008, 22:

Access the most recent version at doi:10.1101/gad.1667708
Related Content Intriguing parasites and intramembrane proteases
Robert B. Rawson
Genes Dev. June , 2008 22: 1561-1566
References This article cites 51 articles, 23 of which can be accessed free at:
http://genesdev.cshlp.org/content/22/12/1636.full.html\#ref-list-1
Articles cited in:
http://genesdev.cshlp.org/content/22/12/1636.full.html\#related-urls
License
Email Alerting
Service $\begin{aligned} & \text { Receive free email alerts when new articles cite this article - sign up in the box at the top } \\ & \text { right corner of the article or click here. }\end{aligned}$

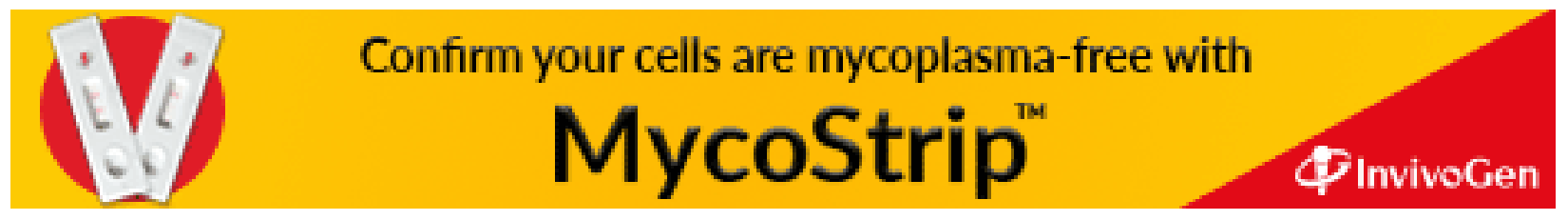

